
$\$$ Research Square
Preprints are preliminary reports that have not undergone peer review.
They should not be considered conclusive, used to inform clinical practice, or referenced by the media as validated information.

\title{
The Value of The Accessory Pathway Potential in Guiding The Electrode Selection for Ablation Within Coronary Sinus
}

\section{XiaoLin Sun}

Northern Jiangsu People's Hospital https://orcid.org/0000-0002-8486-8776

Jiang Jiang

Northern Jiangsu People's Hospital

Pei Xu

Jiangsu Taizhou People's Hospital

Kai Jin

Yangzhou University

ZhengYu Bao ( $\nabla 1403658071 @ q q . c o m$ )

Northern Jiangsu People's Hospital https://orcid.org/0000-0001-9663-411X

\section{Research article}

Keywords: Coronary sinus, Accessory pathway potential, Ablation catheter, Temperature-controlled catheters, Irrigated-tip catheters

Posted Date: October 13th, 2020

DOl: https://doi.org/10.21203/rs.3.rs-88855/v1

License: (c) (i) This work is licensed under a Creative Commons Attribution 4.0 International License. Read Full License 


\section{Abstract}

Background: The ablation of the coronary sinus (CS) is becoming more and more important in accessory pathway-related supraventricular tachycardia ablation. In comparison with temperature-controlled catheters (TCC), irrigated-tip catheter (ITC) are more expensive and cause more damage in accessory pathway-related supraventricular tachycardia ablation. We intended to develop a new method for the reasonable selection of electrode for coronary sinus(CS) ablation via assessing the relationship between the accessory pathway (AP) potential and time of successful ablation.

Methods $₫ A$ mong the patients who had detected the bypass potential during radiofrequency ablation between 1/1/2015 and 12/31/2019. TCC was performed in 330 patients receiving endocardial ablation. 30 patients underwent radiofrequency catheter ablation (RFCA) in CS. The relationship between AP potential and time of successful ablation was analyzed.

Results $\llbracket 330$ cases of TCC ablation within the endocardium revealed that the amplitude of AP potential was significantly and negatively correlated with the time of successful ablation $(r=-0.79, p<0.001)$.

In CS ablation, the median baseline amplitude of the AP potentials in patients with successful TCC ablation was higher than that in patients with ITC following TCC ablation failure $(p=0.02)$. The optimal cutoff value of the amplitude of the AP potential to guide the selection of a catheter for ablation was 1.07 $\mathrm{mV}$, and the sensitivity and specificity were $80 \%$ and $90 \%$, respectively.

Conclusion区The AP potential is helpful for the electrode selection in CS ablation.

\section{Background}

Radiofrequency catheter ablation (RFCA) is currently considered the preferred treatment for patients with accessory pathway-related supraventricular tachycardia (AP-SVT). Previous studies have shown that the success rate for catheter ablation is greater than 95\% [1], and can be even higher when the AP is located in the posterior mitral valve [2]. However, some bypass tracts are near the epicardium of the valve, which is not easy to be ablated within the endocardium pathway. In such situations, ablation within the CS can significantly improve ablation success rates. However, procedure-related complications such as coronary vessel injury and CS thrombosis can be more serious [3,4].

Temperature control catheter (TCC) and irrigated-tip catheter (ITC) are two common options in AP-SVT radiofrequency ablation therapy. Compared with TCC, ITC perfused with cold saline can maintain a lower temperature at the electrode-tissue interface, increase thermal osmosis, and the transmission energy of ablation, thus generate a deeper and larger injury [5]. TCC with 4-mm tips have been adequate to ablate APs located near the endocardium. However, these catheters are insufficient enough for the ablation of APs located near the epicardium. 
Given that ITC is more expensive and traumatic, the conventional process is the first choice TCC, if it fails, then choose ITC. Howbeit, using ITC directly can cause unnecessary myocardial damage and is more costly than using TCC. Using ITC after TCC failure can cause prolonged surgical time and additional myocardial damage. Thus, it is of great significance to find a suitable indicator that can guide catheter selection.

AP potential, generated by atrioventricular bypass conduction, located after A wave and before delta wave, is deemed to have the potential to guide catheter ablation. Generally, the farther away from the bypass, the smaller the AP potential. The AP potential can be recorded in $89 \%$ of patients with AP by ventricular or atrial pacing from the side producing the longer local VA or local AV interval, respectively [6]. In our daily clinical practice, we found that patients with high amplitude of AP potential were easier to attain successful ablation by TCC than those with low amplitude of AP potential. We recorded AP potential by TCC and analyzed the correlation between AP potential and the time of successful ablation to explore its guiding significance in the electrode selection in CS ablation.

\section{Methods}

\section{Study population}

This study retrospectively reviewed the records of 360 patients with AP-SVT who had detectable AP potentials in 1545 patients undergoing catheter ablation from 1/1/2015 to 12/31/2019,In the 360 patients, ablation within the endocardium was applied in 330 and CS ablation in 30 patients. In CS, 15 cases were ablated via TCC successfully, and 15 cases were performed with ITC after TCC ablation failure. Essential information included patient characteristics, ECG and electrophysiological findings were collected. Those with structural heart disease were excluded based on echocardiography, cardiac magnetic resonance imaging, or coronary angiography findings.

The study was conducted in accordance with the declaration of Helsinki and the International Conference on Harmonization Good Clinical Practice Guidelines (ICHGCP). The ethical review board of Hospital approved the study protocol. All patients were given written informed consent.

\section{Electrophysiological study}

All procedures were performed under local anesthesia. Two quadripolar catheters were then placed into each patient: one in the right atrium and another in the right ventricle. In addition, one decapolar catheter was positioned in the CS through a femoral approach. An electrode in the inferior vena cava $(25 \mathrm{~cm}$ from the tip of a right atrial catheter) was used as the reference electrode for unipolar recordings (Ablation electrode is the positive pole; inferior vena cava electrode is the negative pole; and filter settings of 1 to $500 \mathrm{~Hz}$ reduce baseline drift during respiration), and recorded with a digital electrophysiological register system (EP-Work Mate / Work Mate Claris, St.Jude Medical, St.Paul, MN, USA). Programmed electrical stimulation was used to evaluate the atrium, ventricle, AV node, and AP conduction, and induce tachycardia. Tachycardia can also be induced by intravenous isoproterenol (1-4 mg/min), if needed. 


\section{Mapping and recording of accessory pathway potential}

Endocardial and CS mapping were performed to identify optimal target sites for ablation. A 7Fr quadripolar TCC with a 4-mm tip(Cordis Webster Inc., Baldwin Park, CA, USA)was inserted through the right femoral vein and positioned in the tricuspid annulus, or in the mitral annulus via trans-septal puncture, or in CS to map and record AP.

The tricuspid/mitral annulus was mapped via the right femoral vein during tachycardia, as well as fixed ventricular pacing. Ideal ablation targets were those with AP potential or the earliest excited $V$ wave or reverse $A$ wave. If there were no ideal targets after endocardium mapping, or always a distance between A-V/V-A, or the bypass cannot be blocked by ablating at the earliest excited point of V-wave or A-wave. Then the ablation catheter was inserted into the CS or its branch for further mapping. If an ideal target can be found in CS, try to ablate by TCC for 10 seconds, and ITC is performed when the TCC ablation fails.

Electrophysiological register system recorded a sharp potential between $A$ wave and $\mathrm{P}$ wave. When pacing the atria with a fixed-frequency (S1S1), and then stimulating the right ventricle (S1S2) in advance, as $\mathrm{S} 2$ gradually advanced, a sharp potential has nothing to do with $A$ wave, $V$ wave, which is termed as AP potential.

\section{Endocardial and CS Ablation}

Ablation was performed with 4-mm TCC (Cordis Webster Inc., Baldwin Park, CA, USA) or irrigated 4-mm catheter (Thermocool SF, Biosense Webster, Diamond Bar, CA, USA). During endocardium ablation, we used temperature-controlled ablation catheters $\left(40-50^{\circ} \mathrm{C}, 30-40 \mathrm{~W}\right)$. In CS ablation, a radiofrequency application was set with target temperatures of $40-50^{\circ} \mathrm{C}$ and maximum power outputs of $20-30 \mathrm{~W}$ for the non-irrigated catheter and $\left(43^{\circ} \mathrm{C}, 15-25 \mathrm{~W}\right)$ for the irrigated catheter, respectively. Prior to ablation, a weight-adjusted bolus of unfractioned heparin was administered intravenously. And meanwhile, coronary angiography was performed to prevent injury to the coronary artery. Effective ablation will be achieved with a consolidation discharge lasting 60-90 seconds. Ablation success criteria were the disappearance of the delta wave on the surface ECG and elimination of antegrade and retrograde conduction over the AP in the postablation electrophysiological study with an isoproterenol infusion. If the antegrade and retrograde blocks were achieved, wait 30 minutes to evaluate whether the operation was successful.

\section{Blood sample collection and cTnl assay}

Cardiac troponin I (cTnl) levels were measured in all patients 24 hours before and 6 hours after ablation. Plasma was collected after centrifugation, stored at $-80^{\circ} \mathrm{C}$. The amplitudes of the AP potentials were measured using the digital electrophysiological register system. Following surgery, all patients underwent ECG monitoring for at least 24 hours.

\section{Patient follow-up}


After surgery, patients received antithrombotic therapy with aspirin for 1 month. After discharge from the hospital, patients were examined at our arrhythmia clinic or by their referring cardiologists every 3 to 6 months for the first year and annually thereafter.

\section{Statistics}

Continuous variables are presented as the median with the interquartile range. Continuous variables of independent groups were compared using the Mann-Whitney $\mathrm{U}$ test, and comparisons of paired groups were analyzed using the Wilcoxon Signed-Rank test. To assess the predictive performance of the AP potential with respect to catheter selection, a receiver operating characteristics (ROC) curve was plotted. Subsequently, cut-off values of the AP potential were determined based on the Youden index from the ROC curve. A P value less than 0.05 was considered statistically significant. All statistical analyses were performed using SPSS22 (SPSS Inc., Chicago, IL, USA).

\section{Results}

\section{Baseline patient characteristics}

The baseline characteristics of 30 patients undergoing CS ablation are shown in (Fig. 1) and (Table 1). The median age was 41 years in the TCC (IQR 34-36 years; one male patient) and 43 years (IQR 33-37 years; three male patients; $p=0.884)$ in the ITC + TCC groups. The left ventricular ejection fraction was $55 \%$ (IQR 54-56\%) and 56\% (IQR 54-58\%; $\mathrm{p}=0.365$ ), and cTnl levels were $0.21 \mathrm{ng} / \mathrm{mL}$ (IQR 0.16$0.28 \mathrm{ng} / \mathrm{mL}$ ) and $0.22 \mathrm{ng} / \mathrm{mL}$ (IQR $0.15-0.36 \mathrm{ng} / \mathrm{mL} ; \mathrm{p}=0.204$ ) in the TCC and ITC + TCC groups, respectively. After patients undergoing CS ablation by ITC + TCC, three patients had ST-segment elevation and two patients had chest pain, while the other patients had no underlying cardiomyopathy.

Table 1

Baseline characteristics of the patients

\begin{tabular}{|llll|}
\hline & TCC $(\mathbf{n}=\mathbf{1 5})$ & ITC + TCC $(\mathbf{n = 1 5 )}$ & p value \\
\hline Age, years (IQR) & $41(34-46)$ & $43(33-47)$ & 0.884 \\
\hline Male gender, no. & 5 & 6 & 0.365 \\
\hline LVEF, \% (IQR) & $55(54-56)$ & $56(54-58)$ & 0.204 \\
\hline $\begin{array}{l}\text { Baseline cTnl levels, ng/mL (IQR) } \\
\text { Note: Data are presented as median (interquartile range). } n \text { indicates number of patients with the } \\
\text { characteristic }\end{array}$ & $0.21(0.16-0.28)$ & $0.22(0.15-0.36)$ & \\
\hline $\begin{array}{l}\text { available. LVEF: left ventricular ejection fraction. ITC: Irrigated-tip catheter; TCC: temperature- } \\
\text { controlled catheters }\end{array}$ & & & \\
\hline
\end{tabular}

Procedural characteristics 
Procedural characteristics are shown in Table 2. Total ablation time, the number of RF lesions, and X ray time were significantly lower in patients undergoing TCC ablations compared to patients undergoing TCC + ITC ablations.

Table 2

Procedural characteristics of the patients

\begin{tabular}{|llll|}
\hline & TCC $(\mathbf{n}=\mathbf{1 5})$ & TCC + ITC $(\mathbf{n = 1 5 )}$ & P value \\
\hline Total RF time (min) & $82(68-88)$ & $121(97-138)$ & $<0.001$ \\
\hline Number of RF lesions & $4(4-5)$ & $8(7-8)$ & $<0.001$ \\
\hline X ray (min) & $38(35-42)$ & $43(35-45)$ & $<0.001$ \\
\hline Amplitudes of AP potentials $(\mathrm{mV})$ & $1.16(0.8-1.25)$ & $0.74(0.55-0.99)$ & 0.02 \\
\hline Note: Data are presented as median (interquartile range). RF: radiofrequency; AP: accessory pathway; \\
\hline Comparison Of CTnI levels in different groups
\end{tabular}

In the 30 patients who underwent CS ablation, the mean baseline cTnl level was $0.21 \mathrm{ng} / \mathrm{mL}$ (IQR $0.15-$ $0.34 \mathrm{ng} / \mathrm{mL}$ ). Six hours after ablation, cTnl levels rose to $1.7 \mathrm{ng} / \mathrm{mL}(\mathrm{IQR} 1.5-3.0 \mathrm{ng} / \mathrm{mL} ; \mathrm{p}<0.001)$. Comparing these levels after ablation by the different types of catheter, patients in the TCC group had lower cTnl levels than those in the ITC + TCC group [1.5 ng/mL (IQR 1.3-1.6 ng/mL) vs. $2.9 \mathrm{ng} / \mathrm{mL}$ (IQR $2.1-3.68 \mathrm{ng} / \mathrm{mL}$ ); $\mathrm{p}<0.001]$, respectively (Table 3).

Table 3

Changes in cTnl levels $(\mathrm{ng} / \mathrm{mL}$ ) before and after ablation using different catheters in CS

\begin{tabular}{|lllll|}
\hline \multicolumn{3}{|c}{ All patients $(\mathbf{n = 3 0})$} & \multicolumn{2}{c|}{ Different catheters $(\mathbf{n}=\mathbf{3 0})$} \\
\hline & Before & After & TCC $(\mathrm{n}=15)$ & TCC + ITC $(\mathrm{n}=15)$ \\
\hline CTnl & $0.21(0.15-0.34)$ & $1.7(1.5-3.0)$ & $1.5(1.3-1.6)$ & $2.9(2.1-3.68)$ \\
\hline P value & $<0.001$ & & $<0.001$ \\
\hline Note: Data are presented as median (interquartile range). & \\
\hline
\end{tabular}

\section{Association of amplitude of AP potential with the time of successful ablation in endocardium ablation}

330 cases of successful TCC ablation within the endocardium revealed that amplitudes of AP potentials were significantly correlated with the time of successful ablation. Spearman's coefficients of rank correlation (r) was as follows: $r=-0.79 ; p<0.001$ (Fig. 2a).

\section{Comparison of bypass potential amplitudes in different groups}


The amplitudes of AP potentials in patients undergoing TCC ablation were higher than those undergoing ITC ablation after TCC ablation failure [1.16 mV (IQR 0.8-1.25) vs. $0.74 \mathrm{mV}$ (IQR $0.55-0.99) ; p=0.02]$ (Table 2). In CS ablation,the optimal cut-off amplitude of AP potentials to predict whether TCC ablation has been successful was determined by ROC analyses. The mean AUC value of the amplitudes of the AP potentials was $0.870(95 \% \mathrm{Cl} 0.657-1.0)(p=0.023)$. The optimal cut-off amplitude to maximize the sum of the sensitivity and specificity for overall survival was $1.07 \mathrm{mV}$ (Fig. 2b).

\section{Discussion}

In bypass-induced tachycardia, ablation near the endocardium tends to have a higher success rate. However, the extension of the CS myocardial coat through the posterior coronary vein, middle cardiac vein, or diverticulum neck, and its connection to the left ventricular epicardium, can form an epicardial posteroseptal AP, which is not easy to be blocked via an endocardial approach. Therefore, CS ablation has become a common approach choice for bypass ablation. Injuries to the coronary vein is the most intractable complications of CS ablation. In addition, manipulations in the CS may also cause a CS thrombosis [3]. Mao [7] suggested that the relationship between the CS and coronary arteries should be evaluated prior to performing CS ablation to avoid unnecessary damage. We have also reported a case of myocardial injury and pericarditis caused by coronary sinus ablation[8]. Our study demonstrates that cTnI levels are increased in patients after RFCA, consistent with results of previous studies $[9,10]$.

Conventional RF catheters have modest efficacy in ablating substrates located deep within the myocardium. An irrigated RF catheter with a high RF power can be used without increasing the impedance, creating a greater RF lesion volume[11]; this approach has been shown to increase tissue damage by $30-50 \%$ in animal experiments using ITC compared to TCC [12]. The damaged tissue can approach $1 \mathrm{~cm}$ deep and $14 \mathrm{~mm}$ in diameter [13]. Hernandez [14] found that tissue damage caused by $25 \mathrm{~W}$ irrigated catheter ablation was similar to that caused by $50 \mathrm{w}$ conventional catheter ablation. In Patrick's study, using non-irrigated catheters as well as cryoablation, was proved to be comparably safe and effective in CS [15]. We found that in CS ablation, patients in whom TCCs were used had lower serum cTnl levels after ablation than in patients in whom TCC + ITC were used. We think that ITC causes more harm to patients, and patients who fail TCC have underwent more the number of RF applications and duration of RFCA procedure, which make patients undergoing TCC + ITC experience more lesions and larger cumulative lesion area.

APs have been thought to course perpendicular to the AV groove. However, most APs have an oblique course [16]. If we placed a multipolar electrode between the atrium and the ventricle. The electrode spacing is relatively small, and a local potential can be recorded. This potential exists independently of $A$ wave and $V$ wave, which is termed as AP potential. A retrograde AP potential is differentiated from the local atrial and ventricular potentials by use of atrial extrastimuli. A late atrial extrastimulus advances the local atrial potential without advancing the AP potential, differentiating the AP potential from local atrial activation. An earlier atrial extrastimulus advances the AP potential without advancing the local ventricular potential, differentiating the AP potential from local ventricular activation [17]. After bypass 
blockade, the potential disappears without affecting the ventricular or atrial waveform. We found a significant correlation between the AP potential and the time of successful ablation in endocardium. Meanwhile we compared data from patients who had undergone successful ablation using TCC with those who had unsuccessful ablation using TCC. The amplitudes of the AP potentials in the successful group were significantly higher than those in the unsuccessful group, which indicates that the AP potential may be related to the ablation time. In CS, the higher amplitudes of AP potentials was, the higher the success rate of TCC ablation was, and the cutoff value was $1.07 \mathrm{mv}$.

\section{Conclusion}

In patients who were detected AP potential during CS ablation, the higher amplitude of the AP potential, the higher the ablation success rate by TCC. Such a rational selection of ablation catheters by the amplitude of AP can reduce myocardial damage, surgical time, and costs for patients. More studies are needed to ascertain the significance of AP potential in the electrode selection for ablation within CS by including more patients.

\section{Abbreviations}

ITC

Irrigated-tip catheter; TCC:temperature-controlled catheters; RFCA:Radiofrequency catheter ablation; CS:coronary sinus; AP:accessory pathway

\section{Declarations}

\section{Ethics approval and consent to participate}

The study was conducted in accordance with the declaration of Helsinki and the International Conference on Harmonization Good Clinical Practice Guidelines (ICHGCP). The ethical review board of Northern Jiangsu People's Hospital approved the study protocol. All patients were given written informed consent.

\section{Consent for publication}

Not Application.

\section{Competing interests}

The authors declare that they have no competing interests.

\section{Funding}

This work was supported by a grant from the Northern Jiangsu People's Hospital (funding code: fcjs201709). Funding support was mainly used for subject study and publication expense. 
Availability of data and materials

The datasets used and analysed during the current study are available from the corresponding author on reasonable request

\section{Authors' contributions}

X-L.S, J.J, P.X and K.J were involved in concept/design, data analysis/interpretation, drafting the article, statistics, and data collection. Z-Y B was involved in concept/design, critical revision of the article, approval of the article statistics, and secured funding. All authors take public responsibility for the content. All authors have read and approved the final version of the manuscript.

\section{Acknowledgements}

The authors thank the participants in this study and the nurses in the cardiac center who assisted with the research testing.

\section{References}

1. Calkins H, Yong P, Miller JM, Olshansky B, Carlson M, Saul JP, et al. Catheter ablation of accessory pathways, atrioventricular nodal reentrant tachycardia, and the atrioventricular junction: final results of a prospective, multicenter clinical trial. The Atakr Multicenter Investigators Group. Circulation 1999; 99(2):262-270.

2. Blomström-Lundqvist C, Scheinman MM, Aliot EM, Alpert JS, Calkins H, Camm AJ, et al. European Society of Cardiology Committee N-HRS: ACC/AHA/ESC guidelines for the management of patients with supraventricular arrhythmias-executive summary. a report of the American college of cardiology/American heart association task force on practice guidelines and the European society of cardiology committee for practice guidelines (writing committee to develop guidelines for the management of patients with supraventricular arrhythmias) developed in collaboration with NASPEHeart Rhythm Society. J. Am. Coll. Cardiol 2003; 42(8):1493-1531.

3. Skanes AC, Jones DL, Teefy P, Guiraudon C, Yee R, Krahn AD, et al. Safety and feasibility of cryothermal ablation within the mid- and distal coronary sinus. J Cardiovasc Electrophysiol 2004, 15(11):1319-1323.

4. Alazard M, Lacotte J, Horvilleur J, Ait-Said M, Salerno F, Manenti V, et al. Preventing the risk of coronary injury in posteroseptal accessory pathway ablation in children: different strategies and advantages of fluoroscopy integrated 3D-mapping system (CARTO-UNIVU ${ }^{\mathrm{TM}}$ ). J Interv Card Electrophysiol 2018; 52(1):127-135.

5. Telishevska M, Faelchle J, Buiatti A, Busch S, Reents T, Bourier F, et al. Irrigated-tip catheters for radiofrequency ablation of right-sided accessory pathways in adolescents. Pacing Clin Electrophysiol 2017, 40(10):1167-1172. 
6. Otomo K, Gonzakez MD, Beckman KJ, Nakagawa H, Becker AE, Shah N, et al. Reversing thedirection of paced ventricular and atrial wavefronts reveals an oblique course in accessory AV pathways and improves localization for catheter ablation. Circulation 2001; 104(5):550-556.

7. Mao J, Moriarty JM, Mandapati R, Boyle NG, Shivkumar K, Vaseghi M. Catheter ablation of accessory pathways near the coronary sinus: value of defining coronary arterial anatomy. Heart Rhythm 2015; 12(3):508-514.

8. Zheng MF, Wang Z, Bao ZY, Myocardial injury and pericarditis after combined left atrial and coronary sinus ablation in Wolff-Parkinson-White syndrome: a case report. BMC Cardiovasc Disord 2020; 201(1):e13229.

9. Hisazaki K, Hasegawa K, Kaseno K, Miyazaki S, Amaya N, Shiomi Y, et al. Endothelial damage and thromboembolic risk after pulmonary vein isolation using the latest ablation technologies: a comparison of the second-generation cryoballoon vs. contact force-sensing radiofrequency ablation. Heart Vessels 2019; 34(3):509-516.

10. Manolis AS, Vassilikos V, Maounis T, Melita-Manolis H, Psarros L, Haliasos A, et al. Detection of myocardial injury during radiofrequency catheter ablation by measuring serum cardiac troponin I levels: procedural correlates. J. Am. Coll. Cardiol 1999; 34(4):1099-1105.

11. Aryana A, O'Neill PG, Pujara DK, Singh SK, Bowers MR, Allen SL, et al. Impact of irrigation flow rate and intrapericardial fluid on cooled-tip epicardial radiofrequency ablation. Heart Rhythm 2016, 13(8):1602-1611

12. Ruffy R, Imran MA, Santel DJ, Wharton JM. Radiofrequency delivery through a cooled catheter tip allows the creation of larger endomyocardial lesions in the ovine heart. J Cardiovasc Electrophysiol 1995; 6(12):1089-1096.

13. Nakagawa H, Yamanashi WS, Pitha JV, Arruda M, Wang X, Ohtomo K, et al. Comparison of in vivo tissue temperature profile and lesion geometry for radiofrequency ablation with a saline-irrigated electrode versus temperature control in a canine thigh muscle preparation. Circulation 1995; 91(8):2264-2273.

14. Hernández Madrid A, del Rey Sánchez JM, González Rebollo JM, Alvarez Mogollón T, Correa C, Ortiz Chercoles Al, et al. Moro CAnatomopathological and biochemical correlation of lesions produced by standard and irrigated $4 \mathrm{~mm}$ radiofrequency catheters. Rev Esp Cardiol 2000;53(10):1347-1355.

15. Leitz P, K. Wasmer, Kobe.J, Dechering.DG, Frommeyer.G, Güner.F, et al. Remaining challenges in catheter ablation of accessory pathways: rare entity of coronary sinus diverticulum-associated pathways. Clin Res Cardiol 2019; 108(4) : 388-394.

16. Otomo K, Gonzakez MD, Beckman KJ, Nakagawa H, Becker AE, Shah N, et al. Reversing the direction of paced ventricular and atrial wavefronts reveals an oblique course in accessory AV pathways and improves localization for catheter ablation. Circulation 2001; 104(5):550-556.

17. Nakagawa $\mathrm{H}$, Jackman WM . Catheter ablation of paroxysmal supraventricular tachycardia. Circulation 2007 ; 116(21). 2465-78. 


\section{Figures}
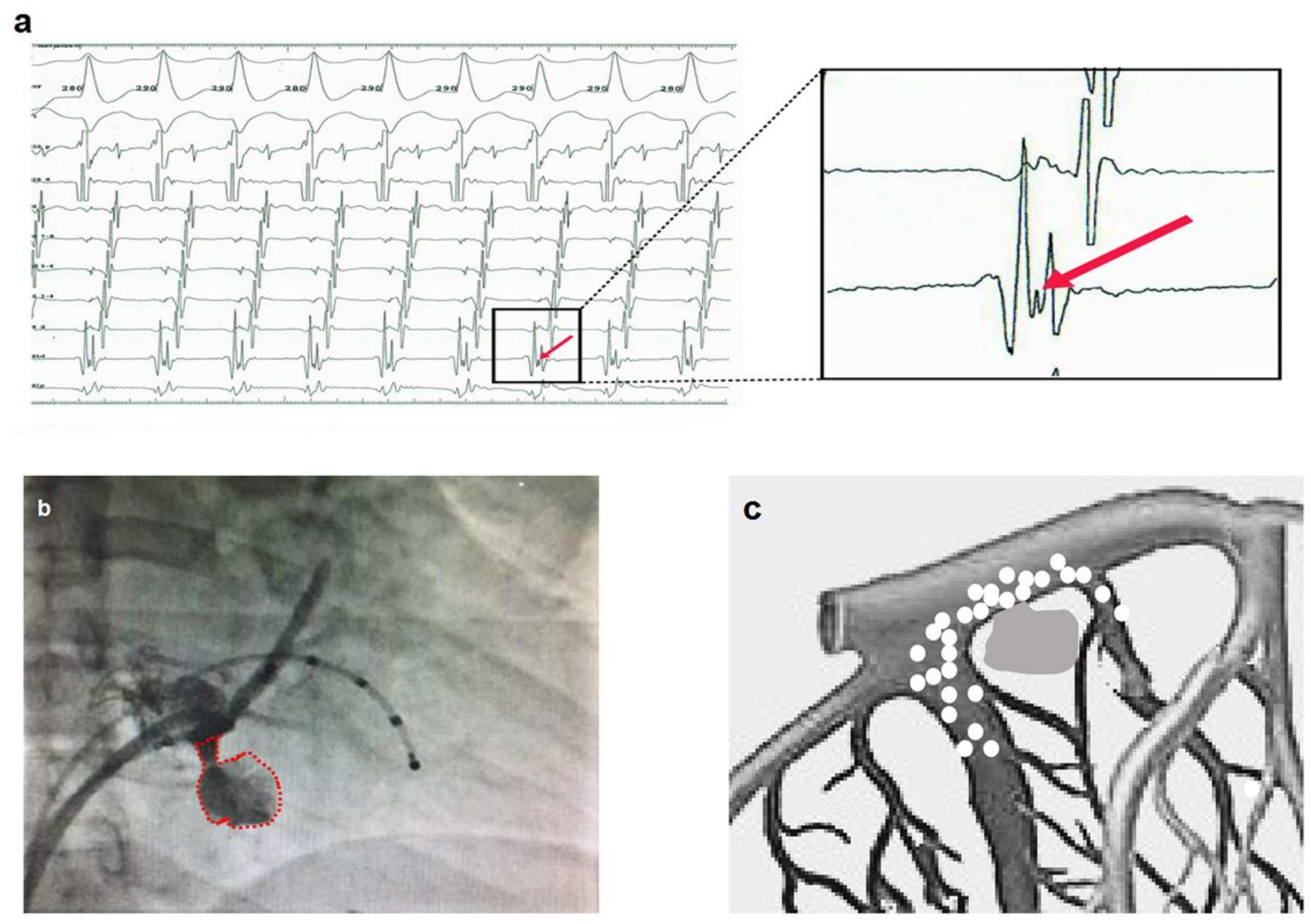

Figure 1

Some characteristics of the patients: (a) The red arrow indicates the accessory pathway potential. (b) Coronary sinus venography shows a diverticulum with a narrow neck (red round dots) near the ostium. (c) The white spots are the locations where 30 cases of ablation in coronary sinus. 
a

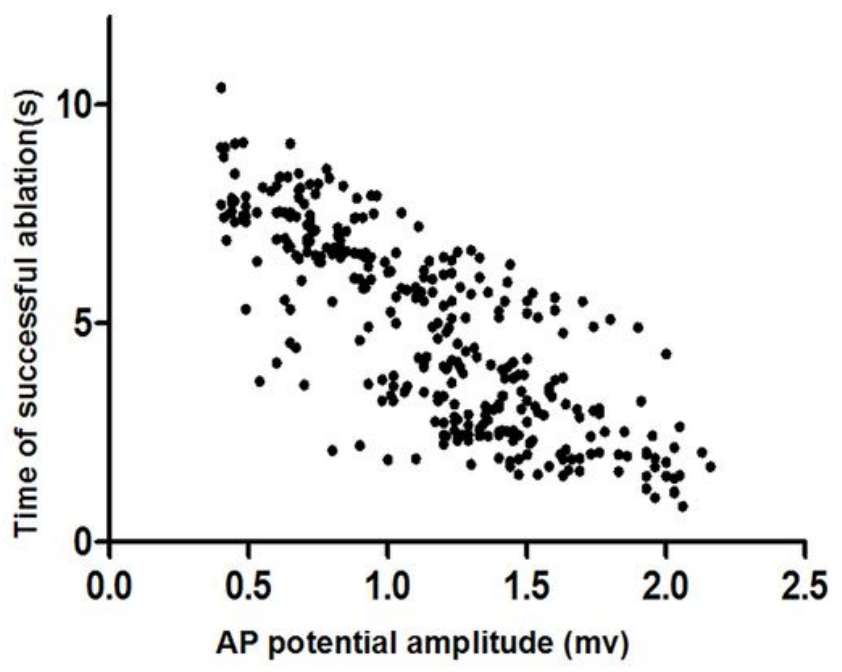

b

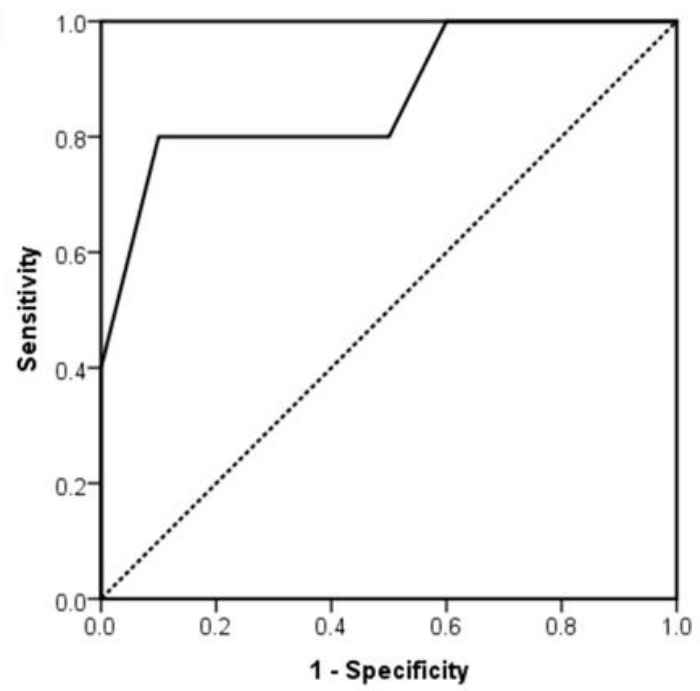

Figure 2

Relationship between AP potential amplitude and the time of successful ablation: (A) Relationship between AP potential amplitude in endocardial and the time of successful ablation were assessed using the Spearman rank correlation coefficient $(p=0.023)$. (b) Receiver operator characteristic curves for predicting which catheter to choose in CS ablation. 